\title{
Problems of Beam Focusing by Electromagnetic Field in Linear Electron Accelerator with Standing Wave, Based on Biperiodic Structure
}

\author{
Aleksandr Evgen'evich Novozhilov ${ }^{1}$, Aleksandr Nikolaevich Filatov ${ }^{1} \&$ Vladimir Kuz'mich Shilov $^{1}$ \\ ${ }^{1}$ National Research Nuclear University "MEPhI", Moscow, Russian Federation \\ Correspondence: Aleksandr Nikolaevich Filatov, National Research Nuclear University "MEPhI", Moscow, \\ 115409, Russian Federation.
}

Received: November 8, 2014

Accepted: November 16, $2014 \quad$ Online Published: January 11, 2015

doi:10.5539/mas.v9n4p160

URL: http://dx.doi.org/10.5539/mas.v9n4p160

\begin{abstract}
In the presented paper problems of particles dynamics in linear electron accelerators with standing wave are discussed. The focus of the study is a possibility of use of focusing properties of electromagnetic accelerating field for passing an electronic beam through aperture of biperiodic slow-wave structure (BSS), which will lead to a significant reduction of an accelerator's sizes. The calculation methodology is proposed and results of the study of particle dynamics in accelerators with standing wave based on BSS are presented, which prove a possibility of beam passing without using external focusing elements. The proposed methodology can be used for a calculation and design of linear electron accelerator (LEA).
\end{abstract}

Keywords: linear electron accelerator, biperiodic slow-wave structure, standing wave, particle dynamics, aperture of passing channel, power lines of electric field, electromagnetic field of cell, passing bushing, range of sustainable radial motion, acceleration range, field of passing channel

\section{Introduction}

An intensive development of accelerator technology for fundamental physical studies in the field of high-energy physics, and a significant progress in other fields of science and technology have provided a possibility for a practical application of accelerators in industry and medicine (Abramyan, 1975; Vahrushin, Gluhih, Nikolaev, \& Rumyantsev, 1995; Vasilyev, Kokontzev, Tyurina, \& Sotnikov, 2011; Gusev, 1976; Zavadtcev, Zavadtcev, Kutzaev, et al., 2011; Komar, 1976).

Accelerators significantly increase efficiency of an industrial production in such fields as: radiation defectoscopy (Nikolaev, Tronov, Rumyantsev, \& Davydov, 1971; Dovbnya, Diki, \& Uvarov, 2000; Auditore, Barna, De Pasquale, et al., 2006), radiation chemistry (Nikolaev, 1971), sterilization of medical products, tools and food products (Vahrushin, Vyaz'menova, Kuznetzov and Fidel'skaya, 1995), element activation analysis (Zavadtsev, Zavadtsev, Kutsaev, et al., 2006; Muntyan, et al., 1979). A large economic effect is achieved in a case of an application of accelerators in geology, in particular, for radiation survey of wells (Bogdanovich, Kaminskiy, Nesterovich, \& Senyukov, 1997).

Accelerators are becoming increasingly widespread in medicine (Agafonov, 1996). Radiation therapy allows to combine a relatively high effectiveness of treatment with a possibility to provide the service for a large number of people.

An interest for linear electron accelerators (LEA) in industry and medicine can be attributed to a number of their advantages. The most important are: easiness of input and output of accelerated particles, which allows to obtain strictly aimed beams of fast electrons and deceleration radiation; easiness of an adjustment of energy and dose rate; high dose rate of deceleration radiation even with a relatively small $(10 \mathrm{MeV})$ energies of accelerated electrons.

In energy range of 2-50 MeV, the most widespread are linear waveguide accelerators with running wave. In a case there is a need for a compact accelerator with a length of waveguide of 0.3-0.5 m, energy of electrons of 4-8 $\mathrm{MeV}$ and small beam current, accelerators with standing wave have a certain advantage (Zavadtsev, Petrov, \& Sobenin, 1994; Sobenin, Yarigin, Kostin, \& Zavadtsev, 1995). In the study (Zavadtsev, 1994,) a comparison of 
regimes of LEA was conducted and it was demonstrated that it is reasonable to develop accelerators with standing wave for small and medium energies.

The analysis of output characteristics of deceleration radiation during defectoscopy and radiation therapy (Belovincev, Bukin, Gaslkevich, et al., 1994) shows, that a large part of problems, solved by use of radiation, in those areas can be solved by using LEA for energy of 4-6 MeV and pulse current of 50-150 mA. Moreover, accelerators, used in defectoscopy and medicine, must be compact and have small longitudinal and transverse dimensions. Reduction of sizes and weight of radiation equipment is, of course, an important and topical task. From the very beginning, the problem was addressed by means of transferring to the three-centimeter wavelength range. Nowadays, a decrease of longitudinal sizes can be achieved by transferring to standing waves mode (Nepp, E., Nepp, B., \& Potter, D., 1968). At the same time, as slow-wave structures in LEA with standing wave BSS are used (Vikulov, Zverev, Zavadcev, et al., 1980), which have high values of specific shunt resistance and gradient of accelerating field.

In general, in BSS a value of aperture for passing of beam is quite small and has a value of about $10 \mathrm{~mm}$ in diameter. Passing of beam through an entire accelerator can be conducted though external focusing of magnetic fields, which can be created by means of using solenoids. Those solenoids can significantly increase transversal dimension of LEA, which is very undesirable from a point of view of using accelerators in defectoscopy and medicine. Thus, one of key problems, appearing during design and development of LEA with standing wave, is a problem of beam passing through BSS aperture. Effectiveness and simplicity of an accelerator's design in a whole in many ways depends on a solution of particle dynamics problem.

As it is well known (Waldner, Vlasov, \& Shal'nov, 1969), in LEA with running wave, particle is gaining energy only on a conditions of synchronous motion with accelerating wave. Because of a relatively low shunt resistance of accelerating structures, working in running waves regime, accelerated particle must be situated in maximum of accelerating field. The constant influence of radial component of electric field, which deflecting it from axis, results in that external focusing elements, particularly, in the beginning of acceleration, had become an integral part of a design of accelerators with running wave. That requires additional energy expenses and makes a design of an accelerator more bulky.

\section{Methodology}

\subsection{General Provisions}

BSS in LEA with standing wave, can be considered as a chain of accelerating resonators, located on a same axis and connected by means connection cells (Negl, E., Nepp, E., \& Nepp, B., 1967). Since cells are free from electromagnetic fields and do not take part in a process of particle acceleration, they can be moved outside of an accelerator's structure. Fluctuations in electromagnetic fields in neighboring accelerated resonators are differ in phase by $\pi$, therefore a particle must transfer through a distance between middles of neighboring resonators for time, which is equal to a half of a period of electromagnetic fluctuations. Only in that case a particle and accelerating fields are synchronized.

If in a fixed point in time we consider a function of a distribution of intensity of electric field along axis of LEA with standing wave, it can be seen that it consists of alternation of pulses of different polarity with a spatial period, equal to a wavelength of a generator.

Using well-known conditions (Vigodski, 2006), such a distribution function can be transformed into Fourier series and present a field of standing wave by sum of harmonics. A particle effectively interacts with that harmonics, which phase speed coincides with a speed of a particle. Consequently, longitudinal particle dynamics in LEA with standing wave can be studied using methods, implemented for accelerators with running wave.

Considering radial movement of particles, the fact must be noted, that longitudinal and radial movements are closely connected and their equations must be solved together. In LEA with standing wave based on BSS, during a calculation of radial movement of particles, certain features can be noted. For resonators, optimized by shunt resistance of complex shape, there is no analytical expression for components of electromagnetic field. Found using numerical methods of distribution of electromagnetic field components by a longitudinal coordinate and by radius, must be defined in a form of tables along all aperture design of passing channel. A presence in accelerating cells of protruding bushings is a reason for a strong curvature of power lines of electric fields, which leads to an appearance of a point of greatest curvature of a transverse electric field with a significant value. Moreover, passing bushings shield a portion of passing channel from electromagnetic field, as a result, in an accelerating structure appear periodically repeated areas free from electromagnetic field.

Thus, for a calculation of radial movement of particles in LEA with standing wave based on BSS, it is necessary 
to create a program, which takes into account those features, which accelerators with standing wave possess. By using such a program, a movement of particles in BSS with an arbitrary distribution of an external magnetic field can be studied. Moreover, it is necessary for the program to take into account forces of intrinsic field of beam, i.e. to solve self-consistent problem. In a case of small energies of beam (which is characteristic for LEA with standing wave) it is considered, that a main contribution in an intrinsic field is made by a field of space charge and an influence of a radiation field can be omitted (Masunov, Morozov, \& Rashikov, 1988). There are two approaches for a solution of that problem: method kinetic equation and method of macro particles. The first method is widely used for a study of processes occurring in plasma (Potter, 1975) and its application for a calculation of a flow of charged particles in accelerators is complicated.

For modeling of a flow of charged particles in LEA it is easier to use method of macro particles (Roshal', 1979). Coulomb field created by an ensemble of macro particles, is found by means of Green's function method (for simple areas and a small number of macro particles), or by means of solving Poisson equation on a grid (Ilyin, 1988). In view of the fact, that LEA with standing wave is characterized by a high rate energy accumulation, and, therefore, a process of forming particles into clouds is faster, than in accelerators with running wave, the method used for a calculation of forces of space charge by an approximation of clouds of particles by evenly charged ellipsoid of revolution (method of macro particles) in a certain circumstances can have serious advantages.

In order to address a problem of passing of beam through BSS, traditional solenoids can be used, but their application leads to a significant increase in transverse dimensions and weight of an entire equipment.

Other known way to transfer beam is a magnetic periodic focusing system (MPFS), which is widely used for transporting low-energy beams (Mokolovski, \& Sushkov, 1972; Kirshtein, Kaino, \& Waters, 1970). For LEA with standing wave use of MPFS is promising, as it significantly reduces weight and dimensions of focusing elements. A design of BSS has an undoubted advantage as compared to regular slow-wave structures in terms of positioning of magnets. Magnets can be positioned in a space occupied by passing bushes, where there is no electric field, and, therefore they don't influence cells characteristics. However, current technical complications, related with a heat removal from permanent magnets, minimize an advantage of MPFS in comparison with solenoids.

\subsection{Particles Dynamics}

The most promising in LEA with standing wave is focusing, in which beam is passing by means of focusing effect of electromagnetic field itself. As has already been stated above, it becomes possible because of appearance of a radial component of electric field in cells BSS. Use of focusing properties of intrinsic high-frequency field of BSS significantly reduces transverse dimensions of accelerating section and reduces weight of the equipment in a whole. Moreover, it becomes unnecessary to use bulky power supplies of external focusing devices, which are creating additional problems during operation of accelerators, which is especially important in a case of an application in fields of geology, medicine and defectoscopy.

Beam dynamics in a linear accelerator is determined by a movement of each particle, which is contained in beam, under an influence of electrical and magnetic fields. If we assume, that a charge of a particle is a point charge and it doesn't have its own magnetic moment (Artzimovich, \& Lukiyanov, 1978), an equation of motion of an individual particle is described by the following expression

$$
\frac{d}{d t}(m \vec{v})=q \vec{E}+q \mu_{0}[\vec{v} \vec{H}]
$$

where: $t$ - time, $q$ and $m$ - charge and weight of a particle, $\vec{v}$ - its speed, $\mu_{0}-$ magnetic constant, $\vec{E}$ and $\vec{H}-$ high-frequency electromagnetic, static, electric and magnetic focusing fields, or field, created by other particles of beam,

Because, in the final analysis, we are interested in a trajectory of particles of beam in an accelerator, then the equation must be converted into a more convenient, for a study of particle dynamics, form, i.e. to express a particle's acceleration through its speed and intensity of electric and magnetic fields. Because speeds of motion of particles in an accelerator are close to light speed, then, by means of using well-known formulas of relativistic mechanics (Landau, \& Lifshitz, 1988), the following can be obtained

$$
\frac{d \vec{v}}{d t}=\frac{q}{m_{0}} \sqrt{1-\frac{v^{2}}{c^{2}}}\left\{\vec{E}+\mu_{0}[\vec{v} \vec{H}]-\frac{1}{c^{2}} \vec{v}(\vec{v} \vec{E})\right\} .
$$

In general, vectors $\vec{E}$ and $\vec{H}$ depend on coordinates and time, and, in a case, when there is no time dependence (a case of static electrical and magnetic fields), the formula remains its form. 
Obtained vector equation is projected on coordinate axis and, thus, system of scalar equations is obtained. In the discussed case, it is more convenient to use cylindrical coordinate system $(\rho, \varphi, z)$, because passing channel and electromagnetic field at the lowest oscillation possess axial symmetry.

$$
\begin{gathered}
\frac{d^{2} z}{d t^{2}}=\frac{q}{m_{0}} \sqrt{1-\frac{v^{2}}{c^{2}}}\left\{E_{z}+\mu_{0}\left(v_{z} H_{\varphi}-v_{\varphi} H_{\rho}\right)-\frac{1}{c^{2}}\left(v_{z} v_{\rho} E_{\rho}+v_{z} v_{\varphi} E_{\varphi}+v_{z}^{2} E_{z}\right)\right\} \\
\frac{d^{2} \rho}{d t^{2}}=\frac{q}{m_{0}} \sqrt{1-\frac{v^{2}}{c^{2}}}\left\{E_{\rho}+\mu_{0}\left(v_{\varphi} H_{z}-v_{z} H_{\varphi}\right)-\frac{1}{c^{2}}\left(v_{\rho} v_{\varphi} E_{\varphi}+v_{z} v_{\rho} E_{z}+v_{\rho}^{2} E_{\rho}\right)\right\}+\frac{v_{\varphi}^{2}}{\rho} \\
\frac{d(\rho \dot{\varphi})}{d t}=\frac{q}{m_{0}} \sqrt{1-\frac{v^{2}}{c^{2}}}\left\{E_{\varphi}+\mu_{0}\left(v_{z} H_{\rho}-v_{\rho} H_{z}\right)-\frac{1}{c^{2}}\left(v_{\rho} v_{\varphi} E_{\rho}+v_{\varphi} v_{z} E_{z}+v_{\varphi}^{2} E_{\varphi}\right)\right\}+\frac{v_{\rho} v_{\varphi}}{\rho}
\end{gathered}
$$

If we transfer to dimensionless coordinates $\xi=\frac{z}{\lambda}, \eta=\frac{\rho}{\lambda}$, dimensionless time $\tau=\frac{c t}{\lambda}$ and relative speeds by coordinates $\beta=\frac{d \xi}{d \tau}, u=\frac{d \eta}{d \tau}, \alpha=\eta \frac{d \varphi}{d \tau}$, and magnetic constant $\mu_{0}$ will be expressed through wave impedance of vacuum $Z_{0}=120 \pi$ and speed of light, i.e. $\mu_{0}=\frac{Z_{0}}{c}$, then the system of equations will take the following form:

$$
\begin{gathered}
\frac{d^{2} \xi}{d \tau^{2}}=\frac{q \lambda}{m_{0} c^{2}} \frac{1}{\gamma}\left\{E_{\xi}+Z_{0}\left(u H_{\varphi}-\alpha H_{\eta}\right)-\left(\beta u E_{\eta}+\beta \alpha E_{\varphi}+\beta^{2} E_{z}\right)\right\} \\
\frac{d^{2} \eta}{d \tau^{2}}=\frac{q \lambda}{m_{0} c^{2}} \frac{1}{\gamma}\left\{E_{\xi}+Z_{0}\left(\alpha H_{\xi}-\beta H_{\varphi}\right)-\left(u^{2} E_{\eta}+u \alpha E_{\varphi}+\beta u E_{\xi}\right)\right\}+\frac{\alpha^{2}}{\eta} \\
\frac{d \alpha}{d \tau}=\frac{q \lambda}{m_{0} c^{2}} \frac{1}{\gamma}\left\{E_{\varphi}+Z_{0}\left(\beta H_{\xi}-u H_{\xi}\right)-\left(u \alpha E_{\eta}+\alpha^{2} E_{\varphi}+\alpha \beta E_{\xi}\right)\right\}-\frac{\alpha u}{\eta}
\end{gathered}
$$

where $\gamma=\frac{1}{\sqrt{1-\beta^{2}-u^{2}-\alpha^{2}}}-$ energy of a particle in units rest energy, $\lambda$ - wave length of a generator.

In the obtained system of equations, the only remained dimensional components are components of electric and magnetic fields, which are easily transformed into dimensionless form by means of the equations:

$$
\begin{gathered}
A_{i}(\eta, \varphi, \xi, \tau)=\frac{q \lambda}{m_{0} c^{2}} E_{i}(\eta, \varphi, \xi, \tau), \\
B_{i}(\eta, \varphi, \xi, \tau)=\frac{q \lambda}{m_{0} c^{2}} Z_{0} H_{i}(\eta, \varphi, \xi, \tau) ;
\end{gathered}
$$

where $\mathrm{i}=\eta, \varphi, \xi$.

Because we discuss a motion of particles in axial-symmetric files, then a relationship between fields and coordinates in the following will not be considered.

Electromagnetic field in a resonator on the lowest oscillation $E_{010}$ has three components, which in dimensionless form are expressed as follows:

$$
\begin{gathered}
A_{\xi}(\eta, \xi, \tau)=\frac{q \lambda}{m_{0} c^{2}} E_{\xi}(\eta, \xi,) \cos (2 \pi \tau)=A_{\xi 0}(\eta, \xi) \cos (2 \pi \tau), \\
A_{\eta}(\eta, \xi, \tau)=\frac{q \lambda}{m_{0} c^{2}} E_{\eta}(\eta, \xi,) \cos (2 \pi \tau)=A_{\eta 0}(\eta, \xi), \\
B_{\varphi}(\eta, \xi, \tau)=\frac{q \lambda}{m_{0} c^{2}} Z_{0} H_{\varphi}(\eta, \xi,) \sin (2 \pi \tau)=A_{\xi 0}(\eta, \xi) \sin (2 \pi \tau) .
\end{gathered}
$$

External magnetic systems creates static magnetic fields, which dimensionless components in a zone adjacent to an axis can be found, using values of field on an axis itself, i.e. presuming, that field in a zone adjacent to an axis is not dependent on radius:

$$
B_{\xi}(\eta, \xi)=\frac{q \lambda}{m_{0} c^{2}} Z_{0} H_{\varphi}(0, \xi,)
$$




$$
B_{\eta}(\eta, \xi)=-\frac{\eta}{2} \frac{q \lambda}{m_{0} c^{2}} \frac{\partial H_{\xi}}{\partial \xi}(\eta, \xi,)
$$

As a result, the equation of a motion of a charged particle can be to expressed by a system of equations in dimensionless quantities

$$
\begin{gathered}
\frac{d^{2} \xi}{d \tau^{2}}=\frac{1}{\gamma}\left\{A_{\xi 0}(\eta, \xi) \cos (2 \pi \tau)+u B_{\varphi 0}(\eta, \xi) \sin (2 \pi \tau)-\alpha B_{\eta}(\eta, \xi)\right. \\
\left.-\beta\left[u A_{\eta 0}(\eta, \xi) \cos (2 \pi \tau)+\beta A_{\xi 0}(\eta, \xi) \cos (2 \pi \tau)\right]\right\}, \\
\frac{d^{2} \eta}{d \tau^{2}}=\frac{1}{\gamma}\left\{A_{\eta 0}(\eta, \xi) \cos (2 \pi \tau)+\alpha B_{\xi}(\eta, \xi)\right. \\
\left.-\beta B_{\varphi 0}(\eta, \xi) \sin (2 \pi \tau)-u\left[u A_{\eta 0}(\eta, \xi) \cos (2 \pi \tau)+\beta A_{\xi 0}(\eta, \xi) \cos (2 \pi \tau)\right]\right\}+\frac{\alpha^{2}}{\eta}, \\
\frac{d \alpha}{d \tau}=\frac{1}{\gamma}\left\{\beta B_{\eta}(\eta, \xi)-u B_{\xi}(\eta, \xi)-\alpha\left[u A_{\eta 0}(\eta, \xi) \cos (2 \pi \tau)+\beta A_{\xi 0}(\eta, \xi) \cos (2 \pi \tau)\right]\right\}-\frac{\alpha u}{\eta} .
\end{gathered}
$$

The resulting system of equations was used as a basis for studies of a motion of particles in accelerators with standing wave. Due to the fact, that electromagnetic field in a resonator of a complex shape is impossible to express analytically, then corresponding components of a field, included in the right part, must be defined in a form of a table. An external magnetic field can have any form and also must be set in a form of a table.

\subsection{Electromagnetic Field of a Resonator}

During a calculation of dynamics of particles in accelerators with standing wave based on BSS, it is necessary to know a distribution of components of electromagnetic field in an aperture passing channel of an entire structure. In turn, that problem is reduced to a calculation of electromagnetic field and tis own parameters for individual cells, constituting BSS.

A good match of experimental results and results of calculation in a case of LEA with standing wave is obtained in a case of taking into account only the lowest oscillations (of type $E_{010}$ ). In a case of a determination of electromagnetic fields in BSS we will assume that there are no current and energy losses inside a volume of a cell. Then, a calculation of high-frequency fields of an individual resonators with axial-symmetry may be reduced to a scalar problem relatively to azimuthal component of magnetic field $\nabla^{2} H_{\varphi}+k^{2} H_{\varphi}=0$ with the boundary conditions: $\frac{\partial H_{\varphi}}{\partial n}=0$ on conducting surfaces and symmetry planes and $H_{\varphi}=0$ on a resonator's axis.

The equation contains two unknown: proper number $k=\frac{2 \pi}{\lambda}$ ( $\lambda$ - wavelength) and magnetic field $H_{\varphi}$, and, therefore, another one must be added, which is variational formulation of the problem $k^{2}=\frac{\int_{V}\left(\nabla H_{\varphi}\right)^{2} d V}{\int_{V} H_{\varphi}^{2} d V}$, where $\mathrm{V}$ - volume of a cell.

A method of solving the problem is finite difference method with double iteration cycle by magnetic field and wave number. In order to create finite-difference scheme, 5 point approximation was used, method of consequent over-relaxation was used for solving that system. Automated application of grid on an unconditioned boundary, defining of boundary point and an automated application of boundary conditions is of a special importance. A solution of that problem allows to considerably decrease time for a preparation for a calculation of a certain resonator and allow to become free of an association with a certain form of a cell.

An algorithm of automated application of a grid and boundary points' determination is based on the fact, that any boundary curve can be approximated with a necessary order of accuracy by segments of lines and arcs of corresponding circles. In a case of real resonators considered by BSS, boundary contour of their longitudinal cross-section is formed from above mentioned fragments. Thus, during setting of geometry of a boundary, it is necessary to specify coefficient in equations of line, radii and centers of circles' arcs and borders of various areas by radial coordinate. Those requirements are meet in almost every used resonator.

An application of a grid on a specified area is carried out from left to right. Key parameters, which completely 
describe applied grid are: number of internal nodes in every line of grid, total number of nodes in line, i.e. sum of internal and external nodes, and number of internal lines of grid. After a determination of those parameters, it's necessary to specify boundary conditions for each external node of the grid. Those conditions are value of slope of normal to a boundary and indexes of nodes, which are present in a boundary equation of that node. If step of node during time of work of program doesn't change, then boundary conditions don't change as well. They are used during a calculation of values $H_{\varphi}$ in boundary nodes. The program has a feature of iteration on a sequence of grids, when a step of a grid is decrease from one number of iterations to another, and densening of grid, when areas of strong change of a field are covered with a denser grid in advance. Those two methods are hard to combine because of an appearance of new nodes on a boundary of areas with different density of grid with simultaneous decrease of its step.

As an initial approximation, a distribution of magnetic field of cylindrical resonator and its wave number are used. Then iteration process by field is conducted and wave number is clarified. A calculation is stopped after achieving a specified accuracy, usually $3 \cdot 10^{-4}$ by wave number, which corresponds to $10^{-2}$ by magnetic field. At the same time, number of iterations by field is remained constant and is about 10 in the beginning of the count and reaching 50 by its end. As about a number of iterations by wave number, it is approximately 25 .

\section{Results}

A principle of focusing by means of electromagnetic field of an accelerator with standing wave will be demonstrated on an example of a single accelerating cell, which is a spacing of BSS with external communication cells. Because of a curvature of power line of electric field in an area of passing channel, in a first half of accelerating gap the field bas a radial component, directed towards an axis, in a second half-directed outwards of an axis. A particle, moving along an axis, upon entering accelerating gap is subjected to force impulse in direction of an axis (focusing), upon exiting - impulse out of an axis (defocussing). An alteration of focusing and defocussing electric field with a certain conditions can lead to a focusing effect (Fainberg, 1962).

Let's determine an area of sustainable radial movement and an area of longitudinal acceleration of a particle for an individual spacing of BSS with external communication cells. A distribution of components of intensity of electromagnetic filed in an area of passing channel, obtained earlier, allows to create a simplified model of a cell, and, using simple analytical expression, to determine an area of phases, in which simultaneous longitudinal acceleration and sustainability of a radial movement of a particle is possible.

First, let's discuss a radial movement of a particle. Presuming, that a particle passes a spacing of BSS with a constant speed $\frac{v_{z}}{c}=\beta_{z} \approx 1$ on a certain distance from $\mathrm{z}$ axis. A distribution of a radial component of intensity of electric field $\stackrel{c}{E_{\rho}}\left(\rho_{0}, z\right)$ along an axis can be expressed analytically on sections of a spacing $D$ :

$$
\begin{array}{cc}
z_{1} \leq z \leq z_{2} & E_{\rho}\left(\rho_{0}, z\right)=\frac{-E_{\rho 0}\left(z-z_{1}\right)}{z_{2}-z_{1}}, \\
z_{2} \leq z \leq z_{3} & E_{\rho}\left(\rho_{0}, z\right)=\frac{-E_{\rho 0}\left(z-z_{3}\right)}{z_{2}-z_{3}}, \\
z_{4} \leq z \leq z_{5} & E_{\rho}\left(\rho_{0}, z\right)=\frac{E_{\rho 0}\left(z-z_{4}\right)}{z_{5}-z_{4}}, \\
z_{5} \leq z \leq z_{6} & E_{\rho}\left(\rho_{0}, z\right)=\frac{E_{\rho 0}\left(z-z_{6}\right)}{z_{5}-z_{6}}, \\
0 \leq z \leq z_{1}, \quad z_{3} \leq z \leq z_{4}, & z_{6} \leq z \leq D \quad E_{\rho}\left(\rho_{0}, z\right)=0 .
\end{array}
$$

For an accelerating cell with maximum shunt resistance, distribution $E_{\rho}\left(\rho_{0}, z\right)$ has a following form:

$$
\begin{gathered}
z_{2}-z_{1}=z_{3}-z_{2}=\frac{\Delta z}{2}=0.05 D, \\
z_{5}-z_{4}=z_{6}-z_{5}=\frac{\Delta z}{2}=0.05 D, \\
z_{2}=D-z_{5}=0.2 D,
\end{gathered}
$$

Upon passing spacing, a particle with charge $q$ will change its radial impulse on a value 


$$
\Delta p_{\rho}=q \int_{0}^{D / v_{z}} E_{\rho}\left(\rho_{0}, z\right) \cos \left(\omega t+\varphi_{0}\right) d t,
$$

where $\varphi_{0}$ - a phase of entering of a particle with $\mathrm{z}=0, \omega-$ a frequency of a generator. As $E_{\rho}$ is in a complex manner related with $z$, then it is easier to transfer to integration by $z$, using a relationship $v_{z} t=z$.Then

$$
\Delta p_{\rho}=\frac{q}{\beta_{z} c} \int_{0}^{D} E_{\rho}\left(\rho_{0}, z\right) \cos \left(\frac{2 \pi}{\lambda \beta_{z}} z+\varphi_{0}\right) d t .
$$

Considering a distribution of $E_{\rho}\left(\rho_{0}, z\right)$ along an axis $z$

$$
\begin{aligned}
\Delta p_{\rho}=\frac{2 E_{\rho 0} q}{\Delta z \beta_{z} c}[- & \int_{z_{1}}^{z_{2}}\left(z-z_{1}\right) \cos \left(\frac{2 \pi}{\lambda \beta_{z}} z+\varphi_{0}\right) d z-\int_{z_{1}}^{z_{2}}\left(z-z_{3}\right) \cos \left(\frac{2 \pi}{\lambda \beta_{z}} z+\varphi_{0}\right) d z \\
& \left.+\int_{z_{4}}^{z_{5}}\left(z-z_{4}\right) \cos \left(\frac{2 \pi}{\lambda \beta_{z}} z+\varphi_{0}\right) d z+\int_{z_{5}}^{z_{6}}\left(z-z_{6}\right) \cos \left(\frac{2 \pi}{\lambda \beta_{z}} z+\varphi_{0}\right) d z\right],
\end{aligned}
$$

and after simple transformations, a change of radial impulse of a particle upon passing spacing $D$ will have a following form

$$
\Delta p_{\rho}=\frac{2 E_{\rho 0} q}{\Delta z \beta_{z} c} \frac{\beta_{z}^{2}}{\pi^{2}}\left[1-\cos \left(\frac{2 \pi}{\lambda \beta_{z}} \frac{\Delta z}{2}\right)\right]\left[\cos \left(\frac{2 \pi}{\lambda \beta_{z}} z_{2}+\varphi_{0}\right)\right] .
$$

Area of phases $\varphi_{0}$, where the last expression will be less than 0 , will be considered an area of a sustainable radial movement. Then, taking into account a distribution of a radial component of electric filed along axis $z$, a condition of a sustainable radial movement may be expressed in the final form

$$
\sin \left(\frac{\pi}{2}+\varphi_{0}\right)>0
$$

The last inequality determines an interval of $\operatorname{phases} \varphi_{0}$, where radial movement is sustainable, i.e. $\Delta p_{\rho}<0$ :

$$
-\frac{\pi}{2} \mp 2 \pi k<\varphi_{0}<\frac{\pi}{2} \mp 2 \pi k, \text { where } k=0,1,2 \ldots
$$

Let's consider only longitudinal movement and determine an area of a longitudinal acceleration. A distribution of intensity of accelerating field $E_{z}(z)$ along length of spacing of a structure is presented in fig..

Expressing a change of longitudinal impulse of a particle upon passing of spacing $D$

$$
\Delta p_{z}=q \int_{0}^{D / v_{z}} E_{z}(z) \cos \left(\omega t+\varphi_{0}\right) d t .
$$

Because $E_{z}(z)=E_{z 0}=$ const for $z_{2}<z<z_{5}$ and $E_{z}(z)=0$ for other sectors of spacing $D$, then in the last equation it is possible to not transfer to an integration by, then

$$
\Delta p_{z}=q E_{z 0} \int_{z_{2} / v_{z}}^{z_{5} / v_{z}} \cos \left(\omega t+\varphi_{0}\right) d t
$$

Or

$$
\Delta p_{z}=\frac{q E_{z 0}}{\omega} 2 \sin \left(\omega \frac{z_{5}-z_{2}}{2 v_{z}}\right) \cos \left(\omega \frac{z_{5}-z_{2}}{2 v_{z}}+\varphi_{0}\right) .
$$

The condition of longitudinal acceleration

$$
\Delta p_{z}=\frac{q E_{z 0}}{\omega} 2 \sin (0.3 \pi) \cos \left(\frac{\pi}{2}+\varphi_{0}\right)>0
$$

Or

$$
\cos \left(\frac{\pi}{2}+\varphi_{0}\right)>0
$$

The last inequality determines an interval of phasescos $\left(\frac{\pi}{2}+\varphi_{0}\right)>0$, where particles' acceleration takes place

$$
-\pi \mp 2 \pi k<\varphi_{0}<0 \mp 2 \pi k .
$$




\section{Discussion}

If we compare an interval of particles' acceleration with the interval obtained earlier for a radial movement, it is clear, that phase area of sustainable radial movement and longitudinal acceleration are shifted relatively to each other on $\frac{\pi}{2}$.Common for both areas is the integral

$$
-\frac{\pi}{2} \mp 2 \pi k<\varphi_{0}<0 \mp 2 \pi k .
$$

In limits of that range of measuring $\varphi_{0}$ there are conditions of sustainable radial movement and acceleration of particles. Thus, maximally allowed phase extent of cloud of particles during an injection in accelerating sections must not exceed $\frac{\pi}{2}$.

It is obvious, that boundary points of common range are corresponding to two different regimes: $\varphi_{0}=-\frac{\pi}{2}-$ phase of the largest accumulation of energy by particles, $\varphi_{0}=0-$ phase, corresponding to the fullest use of focusing properties of electromagnetic field of a cell. Naturally, injecting a cloud of particles in those phases is undesirable, because of a risk to lose the major part of particles during further acceleration. That's why an optimal phase of injection is one that satisfies the last inequality. Depending in conditions, which are necessary to be realized in an each case (it is whether maximum accumulation of energy or maximum us of focusing properties of electromagnetic field), a phase of an injection of a cloud must be selected closer to one or another boundary of a limiting interval.

In order to clarify possibilities for focusing by means of electromagnetic field, a numerical integration was carried out of equations of motion with various beginning phases of entering of a particles, which moves in parallel to an axis on an entrance to a real cell of BSS.A particle's trajectory is deflected in a different way, depending on a phase of entering: for one types of phases of entering, entering trajectory is deflected from an axis, for others a cell is similar to a focusing lens. A detailed analysis of calculation results show, that in a case of particles with entering phases $1.45 \pi<\varphi_{0}<2.45 \pi$, a cell is acting as a focusing lens, at that, the biggest focusing effect is achieve in a case of $\varphi_{0}=2.04 \pi$, , and maximum accumulation of energy by a particle is corresponding to entering phase $\varphi_{0}=1.45 \pi$.

It is necessary to note, that a value of intensity of electromagnetic accelerating field in a cell doesn't influence a size and a position of areas of sustainable radial movement and acceleration. In the same way as in a case of an analytical study of properties of accelerating field of a cell, obtained areas are shifted on $\frac{\pi}{2}$ and their extent corresponds with results obtained earlier. An increase of an extent of an accelerating field significantly strengthens focusing action of a cell for entering phases in $\operatorname{area} \varphi_{0}=2.04 \pi$, i.e. in a place, where acceleration of particles is almost zero. For phases in area $\varphi_{0}=1.45 \pi$ (area of maximum accumulation of energy), strengthening of focusing properties of a cell doesn't occur. That fact shouldn't be ignored during an implementation of focusing properties of electromagnetic field in structures based on BSS.

Results of calculation of a particle, entering a cell and parallel to an axis on various distances from it shows, that the closer particle is to an axis, the less obvious is focusing action of electromagnetic field of a cell.

It is related with a fact, that power line of electric field have the highest curvature in an area of passing bushings and the smallest curvature is near an axis.

The most interesting is a case, when a particle is entering into a cell at a negative angle, which corresponds to beam converging at an entrance. In that case, with an initial angle $-1^{\circ}$, an area of radial sustainability is increasing on $0.4 \pi$ and starts to comprise an area of maximum acceleration. It allows to use a regime of maximum acceleration without decreasing radial characteristics of beam. It should be noted, that an obtainment of beams with a negative convergence to 1 degree is not a difficult problem, and use of that kind of beams allow to fully use focusing properties of electromagnetic fields of BSS.

All above mentioned is true also for a case, when a speed of particle is less than a speed of light, but in that case it necessary to consider effect of an increase of speed of a particle upon passing a cell. It leads to a situation, when a particle, with other conditions being equal, is in an area of defocusing electric field for less time, which gives an additional focusing effect. An additional focusing action of electromagnetic field with $\beta_{z}<1$ can be effectively used in an initial stage of acceleration in order to form beam by radius.

\section{Conclusion}

The proposed method of passing beam of electrons in linear accelerators is based on an implementation of focusing properties of radial component of electric field. That component appears even in a case of the lowest type of oscillations in a regular cylindrical resonator in an area of passing tubes, and, therefore, near an axis of 
passing channel. That kind of effect, appearing due to a curvature of power lines of electromagnetic field, can be a prospective solution for proton and ion accelerators (Vdovin, Demchenko, Gusev, Shulika and Shulika, 2010).

Studies, conducted with use of mathematical simulation, demonstrated prospective of that method for research and development of LEA without using of external focusing devices. It allows to considerably reduce dimensions and weight of an accelerator and to increase efficiency in industrial and medical applications.

In further, it is proposed to study in detail an influence of changes of shape of resonators of BSS on a distribution of components of electromagnetic field in an area of passing channel conditions of effective acceleration and stable radial sustainability:

- change of diameter of passing channel for an increase of efficiency of acceleration and beam focusing;

- influence of drift tube length on acceleration rate;

- influence of geometrical shape of drift tubes on radial sustainability of beam.

Author would like to express their gratitude for all people who took part in discussing of that paper.

Thanks. "The article was prepared with the support of the Head of the Department" Electrical "MEPhI (National Research Nuclear University" Moscow Engineering Physics Institute "), we thank professor Schoolkov Edward Jakovljevic and professor of electrophysical installations "MEPhI Sobenina Nicholaya Pavlovicha.

\section{References}

Abramyan, E. (1975). Prospects of an implementation of relativistic electron beams in industrial technology. Bulletin of Academy of Sciences of USSR, 1, 57-68.

Agafonov, A. (1996). Accelerators in medicine. $15^{\text {th }}$ conference on charged particles' accelerators, Protivino, 2 , 366-373.

Artzimovich, L., \& Luk'yanov, S. (1978). Movement of charged particles in electrical and magnetic fields. Moscow: Science.

Auditore, L., Barna, R., \& De Pasquale, D., et al. (2006). A Compact 5 MeV, S-Band, Electron Linac Based $\mathrm{X}$-Ray Tomography System. Proceeding conference EP AC'06, Edinburgh, Scotland.

Belovintzev, K., Bukin, A., \& Gaskevich, E., et al. (1994). Radiation complex for fundamental and applied research. $14^{\text {th }}$ conference on charged particles' accelerators, Protivino, 4, 264-268.

Bogdanovich, B., Kaminskiy, V., Nesterovich, A., \& Senyukov, V. (1997). Small-sized electron linear RF accelerator with beam auto acceleration for geology and industry. Bulletin of American Physical Society, 3(42), 1376.

Dovbnya, A., Diki, N., \& Uvarov, V. (2000). On a possibility of a production of isotopes for nuclear medicine in electron accelerator. $17^{\text {th }}$ conference on charged particles' accelerators, Protivino, 2, 400-404.

Fainberg, Ya. (1962). On a possibility of simultaneous radial a phase sustainability in linear accelerators without special focusing devices. Theory and calculation of linear accelerator. Moscow: Gosatomizdat.

Gusev, O. (1976). Implementation of charged particles accelerators in economy. Reports of second All-Soviet-Union conference on charged particles accelerators, Volume 1. Moscow: Science.

Ilyin, V. (1988). Numerical methods for solution of problems of Electrophysics. Moscow: Science.

Kirshtein, P., Kaino, G., \& Waters, U. (1970). Formation of electron. Moscow: Mir.

Komar, E. (1976). Implementation of charged particles accelerators in economy. Reports of second All-Soviet-Union conference on charged particles accelerators, Volume 1. Moscow: Science.

Landau, L., \& Lifshitz, E. (1988). Theoretical physics. Field theory, Vol. 2. Moscow: Science.

Masunov, E., Morozov, V., \& Rashikov, V. (1988). Calculation of field of space charge of beam in periodic waveguide. Issues of nuclear science and technology, Series: Technology of physical experiment, Kharkov, 2(37), 55-57.

Molokovski, S., \& Sushkov, A. (1972). Intensive electron and ion beams. Moscow: Energy.

Muntyan, V., Prudnikov, I., Shahov, V., Vursnitchenko, S., Zanoshkin, B., Garnik, D., Kon'kov, N., \& Feoktistov, Yu. (1979). Radiation complex based on high-current linear electron accelerators for radiation studies and activation analysis. Reports of Third All-Soviet Union conference on an application of charged particles' accelerators in economy, Leningrad, 1, 86-91. 
Negl, D., Nepp, E., \& Nepp, B. (1967). Description of section of accelerator with standing wave using a model of connected oscillators. Equipment for scientific researches, 11(38), 22-26.

Nepp, E., Nepp, B., \& Potter, D. (1968). Accelerating structures with standing wave for linear accelerator for big energies. Equipment for scientific researches, 7(39), 31-43.

Nikolaev, V. (1971). Linear electron accelerator for sterilization and radiation chemistry. Proceedings of All-Soviet Union scientific and technical conference on an implementation of accelerators in economy and medicine, Leningrad.

Nikolaev, V., Tronov, B., Rumyantzev, V., \& Davidov, V. (1971). Linear electron accelerators for defectoscopy. Proceedings of All-Soviet Union scientific and technical conference on an implementation of accelerators in economy and medicine, Leningrad.

Potter, D. (1975). Computational methods in physics. Moscow: Mir.

Roshal', A. (1979). Modeling of charged beams. Moscow: Atomizdat.

Sobenin, N., Yarigin, S., Kostin, D., \& Zavadtsev, A. (1995, May 1-5). A Biperiodic Accelerating Structure for a Free Electron Laser Buncher. Partical Accelerator Conference and International Conference on High-Energy Accelerators, Dallas, Texas.

Vahrushin, Yu., Gluhih, V., Nikolaev, V., \& Rumyantzev, V. (1995). Development of series of linear electron accelerators for an application in medicine and industry. Synopsizes of report of $8^{\text {th }}$ conference on an application of accelerators of charged particles in industry and medicine, "Accelerators-95", Saint-Petersburg.

Vahrushin, Y., Vyaz'mentzova, G., Kuznetzov, V., \& Fidel'skaya, R. (1995). "Disinfection of waste waters of infectious diseases hospitals by electron beam". Synopsizes of report of $8^{\text {th }}$ conference on an application of accelerators of charged particles in industry and medicine, "Accelerators-95", Saint-Petersburg.

Val'dner, O., Vlasov, A., \& Shal'nov, A. (1969). Linear accelerators. Moscow: Atomizdat.

Vasil'ev, V., Kokontzev, A., Tyurina, S., \& Sotnikov, V. (2011). Large-field radiation exposure of skin by electrons. Medical physics, 4(52), 11-19.

Vdovin, S., Demchenko, P., Gusev, E., Shulika, N., \& Shulika, O. (2010). Combined focusing in linear ion accelerator. Problems of nuclear science and technology, 4(7), 325-329.

Vigodski, M. (2006). Handbook on higher mathematics. Moscow: AST, Astrel'.

Vikulov, F., Zverev, B., \& Zavadtzev, A., et al. (1980). Small-sized linear electron accelerator with standing wave and bridge power circuit. Accelerators, 19. Moscow: Atomizdat.

Zavadtsev, A., Zavadtsev, D., \& Kutsaev, S., et al. (2006). Compact Electron Linear Accelerator Relus-5 for Radiation Technology Application. Proceedings of conference EPAC'06, Edinburgh, Scotland.

Zavadtzev, A., Zavadtzev, D., \& Kutzaev, S., et al. (2011) System of cargo inspection on a basis of dual linear electron accelerator. Devices and equipment for experiment, 2, 151-159.

Zavadtsev, A. (1994). Biperiodic U-structures for Particle Accelerators. Fourth European Accelerator Conference, London, 3, 2176-2178.

Zavadtsev, A., Petrov, Yu., \& Sobenin, N. (1994). Standing Wave Electron LINAC Accelerating Structure for Technology Purposes. Fourth European Accelerator Conference, London, 3, 2173-2175.

\section{Copyrights}

Copyright for this article is retained by the author(s), with first publication rights granted to the journal.

This is an open-access article distributed under the terms and conditions of the Creative Commons Attribution license (http://creativecommons.org/licenses/by/3.0/). 